\title{
Mathematical model of laser radiation femtosecond interaction with human skin
}

\author{
Pavel Rogov ${ }^{*}$, and Viktor Bespalov \\ Saint-Petersburg National Research University of Information Technologies, Mechanics, and Optics, Kronverkskii \\ Prospekt str. 49, Saint-Petersburg, 197101, Russia \\ *e-mail: RogovPU@niuitmo.ru
}

\begin{abstract}
We present a mathematical model of linear and nonlinear processes that occur under the action of femtosecond laser radiation on the cutaneous covering. The analysis is carried out and the analytical solution of the set of equations describing the dynamics of the electron and atomic subsystems is obtained. The results of the work can be used to determine the maximum acceptable energy, generated by femtosecond laser systems, and to develop Russian laser safety standards for femtosecond laser systems. (C) 2016 Journal of Biomedical Photonics \& Engineering.
\end{abstract}

Keywords: laser safety, femtosecond radiation, self-focusing, skin.

Paper \#2847 received 2015.12.30 revised manuscript received 2016.03.01; accepted for publication 2016.03.01; published online 2016.03.29. doi: 10.18287/JBPE16.02.010301

\section{References}

1. S. A. Akhmanov, V. A. Vysloukh, and A. S. Chirkin, Optics of femtosecond laser pulses, Moscow, Nauka (1988) [in Russian]. ISBN 5-02-013838-X.

2. C. Rulliere, Femtosecond laser pulses, Springer (2005). ISBN: 978-0-387-01769-3.

3. B. S. Yilbas, and A. F. M. Arif. "Laser short-pulse heating with time-varying intensity and thermal stress development in the lattice subsystem," Proceedings of the Institution of Mechanical Engineers, Part C: Journal of Mechanical Engineering Science 219(1), 73-81 (2005).

4. L. Habbema, R. Verhagen, R. Van Hal, Y. Liu, and B. Varghese, "Minimally invasive non-thermal laser technology using laser-induced optical breakdown for skin rejuvenation,” Journal of Biophotonics 5(2), 194199 (2011).

5. "GOST 50723-94: Laser Safety. General safety requirements for time-processing and operation of laser products," Moscow, Standards Publishing House (1995) [in Russian].

6. V. Sriramoju, and R. R. Alfano, "In vivo studies of ultrafast near-infrared laser tissue bonding and wound healing," Journal of Biomedical Optics 20(10), 108001 (2015).

7. A. E. Pushkareva, "Methods of mathematical modeling in tissue optics," SPb, ITMO, (2008) [in Russian].

8. I. Fredriksson, M. Larsson, and T. Strömberg, "Optical microcirculatory skin model: assessed by Monte Carlo simulations paired with in vivo laser Doppler flowmetry," Journal of Biomedical Optics 13(1), 014015 (2008).

9. P. Stampfli, and K. H. Bennemann, "Theory for the instability of the diamond structure of Si, Ge, and C induced by a dense electron-hole plasma," Physical Review B 42(11), 7163-7173 (1990).

10. P. Stampfli, and K. H. Bennemann, "Dynamical theory of the laser-induced lattice instability of silicon," Physical Review B 46(17), 10686-10692 (1992).

11. M. F. Kropman, "Dynamics of Water Molecules in Aqueous Solvation Shells," Science 291(5511), 2118-2120 (2001).

12. M. F. Kropman, H.-K. Nienhuys, and H. J. Bakker, "Real-Time Measurement of the Orientational Dynamics of Aqueous Solvation Shells in Bulk Liquid Water," Physical Review Letters 88(7), 077601 (2002).

13. V. P. Lipp, D. S. Ivanov, B. Rethfeld, and M. E. Garcia, "On the interatomic interaction potential that describes bond weakening in classical molecular-dynamic modelling," Journal of Optical Technology 81(5), 254-255 (2014).

14. R. V. Dyukin, G. A. Martsinovskiı̌, G. D. Shandybina, E. B. Yakovlev, I. D. Nikiforov, and I. V. Guk, "Dynamics of the permittivity of a semiconductor acted on by a femtosecond laser," Journal of Optical Technology 78(8), 558-562 (2011). 
15. V. A. Serebryakov, E. V. Boiko, A. G. Kalintsev, A. F. Kornev, A. S. Narivonchik, and A. L. Pavlov "Laser mid-infrared spectral range for precision surgery," Opt J 82(12), 3-13 (2015).

16. I. V. Meglinski, and A. V. Doronin, "Monte Carlo modeling for the needs of biophotonics and biomedical optics," in Advanced Biophotonics: tissue optical sectioning, V. V. Tuchin, and R. K. Wang (eds.), Taylor \& Francis (2012).

17. S. Jacques, and L. Wang, "Monte Carlo modelling of light transport in tissue," in Optical-thermal response of laser-irradiated tissue, A. J. Welch, M. J. C. van Gemert (eds.), Plenum Press, New York (1996).

\section{Introduction}

Femtosecond laser systems (FLSs) are widely used in science, technical applications and medical technologies $[1,2]$. In spite of the fast development of femtosecond technologies and their application in biology and medicine, at present in the Russian Federation there are no standards of safe energy levels of high-intensity femtosecond laser radiation. Note that the mechanism of interaction of intense femtosecond laser radiation with matter essentially differs from that for the pulses of longer duration, since the interaction time is smaller than the time, necessary to excite the phonon subsystem [3]. Besides, the high power density that arises when using the FLSs leads to various nonlinear processes, such as self-focusing, two- and three-photon absorption, multiphoton and impact ionization, and for the high energy density to the optical breakdown. [4]. This process is of particular importance in the case of femtosecond radiation incident on biological tissues, since the high-intensity femtosecond radiation may be essentially dangerous for skin, vision, and other human organs [5].

In the present work, we consider the linear and nonlinear interaction of femtosecond laser radiation with skin and propose a mathematical model approximately describing particular interaction processes. In future, the model can be used to determine the maximal acceptable levels of FLS energy.

\section{Interaction mechanism}

\subsection{Specific features of ultrashort-pulse lasers and the skin}

The human skin is an optically turbid opaque medium (both the absorption and the scattering are present). At the same time, the skin is a heterogeneous structure containing the inclusions of different type and dimension (blood vessels, hair follicles, etc.), which essentially complicates the understanding of the processes that occur under the action of laser radiations on the cutaneous covering. The main elements of the skin are water $(70 \%)$ and proteins $(27 \%)$, and the main structure protein is collagen (nearly $70 \%$ of dry skin weight). The water molecules can be divided into two groups, the free ones and the ones included in protein compounds (bound state, e.g., the three-fold screw group of collagen binds nearly 500 water molecules [6]). Thus, in the first approximation the skin may be considered as water with protein inclusions close to dielectric materials in their electrodynamic properties.
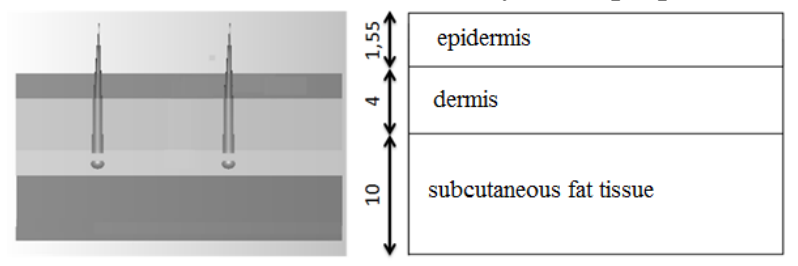

Fig. 1 Model of human skin.

Table 1 The mean optical $(\lambda=800 \mathrm{~nm})$ and thermal physical parameters used in the skin model for calculating [7].

\begin{tabular}{llllllll}
\hline Tissue & $\mu \mathrm{a}, 1 / \mathrm{mm}$ & $\mu \mathrm{s}, 1 / \mathrm{mm}$ & $\mathrm{g}$ & $\mathrm{n}$ & $\mathrm{C}, \mathrm{J} / \mathrm{g} \cdot \mathrm{K}$ & $\mathrm{k}, \mathrm{W} / \mathrm{m} \cdot \mathrm{K}$ & $\mathrm{d}, \mathrm{mm}$ \\
\hline Epidermis & 0.083 & 21.37 & 0.85 & 1.5 & 3.05 & 0.29 \\
Dermis & 0.026 & 9.67 & 0.86 & 1.5 & 3.52 & 0.41 \\
Subcutaneous fat & 0.025 & 12.39 & 0.86 & 1.5 & 2.3 & $0.5-5$ \\
\hline
\end{tabular}

Here $\mu \mathrm{a}$ is the absorption coefficient, $\mu \mathrm{s}$ is the scattering coefficient, $\mathrm{g}$ is the mean cosine of the scattering angle, $\mathrm{n}$ is the mean refractive index, $\mathrm{C}$ is the heat capacity, $\mathrm{k}$ is the heat conduction coefficient, $\mathrm{d}$ is the layer depth.

For estimate, we have chosen the wavelength 800 nm typical for the most widely used titanium-sapphire FLSs. The pulse duration in such systems can vary from 25 to $550 \mathrm{fs}$. The irradiation with ultrashort laser pulses leads to damages that cannot be explained by the thermal melting mechanism [8] and are due to the excitation of valence electrons by the laser pulses [9, 10]. The water molecules serve as the transmitting element that absorb the laser pulse energy and excite the vibrational (phonon) modes of collagen with the relaxation time of the order of 3 ps $[11,12]$.

\subsection{Mathematical model}

In the calculations, we used the set of balance equations that describe the dynamics of electron-atom subsystem. In the model the atoms interact via the semiempirical potentials and the electron degrees of freedom are not taken into account explicitly. The laser radiation generates non-equilibrium charge carriers described by the integral concentration [13]. In this case one can neglect the processes of Auger recombination and 
impact ionization, since for the chosen parameters of radiation their semiempirical contribution is negligibly small.

E.B. Yakovlev et al. [11] proposed the idea of determining the dependence between the potentials of the atoms and the state of the electronic subsystem, thus making it possible to simulate the effects of heat transfer under the action of femtosecond pulses on dielectric materials. The differential Bouguer-Lambert law determines the distribution of the laser radiation intensity $J(z, t)$ inside a solid. The system of heat transfer equation that describes the dynamics of electron and atom subsystems in the one-dimensional approximation has the form:

$$
\begin{aligned}
& \frac{\partial T_{e}}{\partial t}=\alpha_{e} \frac{\partial^{2} T_{e}}{\partial z^{2}}-\frac{1}{C_{e} \tau_{e p}}\left(T_{e}-T_{a}\right)+\frac{\alpha_{e} h v}{C_{e}} J(t, z) \\
& \frac{\partial T_{a}}{\partial t}=\alpha_{a} \frac{\partial^{2} T_{a}}{\partial z^{2}}+\frac{1}{C_{a} \tau_{e p}}\left(T_{e}-T_{a}\right)
\end{aligned}
$$

where $J(t, z)$ is the intensity distribution in the solid, $C_{e}=\frac{\pi^{2} k_{B}^{2} N_{e}}{2 E_{F}}$ is the heat capacity of the electron gas, $C_{a}$ is the heat capacity of atoms, $\tau_{e p}$ is the time of electronphonon relaxation, $T_{e}$ is the temperature of electrons, $T_{a}$ is the temperature of atoms, $\alpha_{e}$ and $\alpha_{a}$ is the thermal diffusivity of electrons and ions; $E_{F}$ is the Fermi energy; $K_{B}$ is the Boltzmann constant.

For modelling the impact of the femtosecond laser radiation on the skin we used the following boundary conditions:

$$
\left.T_{e}\right|_{t=0}=\left.T_{e}\right|_{t=0}=T_{0}
$$

$$
\left.\frac{\partial T_{e}}{\partial z}\right|_{z=0}=\left.\frac{\partial T_{e}}{\partial z}\right|_{z=L}=\left.\frac{\partial T_{a}}{\partial z}\right|_{z=0}=\left.\frac{\partial T_{a}}{\partial z}\right|_{z=L}=0
$$

Since the pulse duration is negligibly small $\left(a_{e} \ll \frac{x^{2}}{t}, a_{a} \ll \frac{x^{2}}{t}\right.$, where $x$ is the spatial representation of the pulse, and the heat diffusivity for a tissue $\alpha_{a}=1.54 \times 10^{-3} \mathrm{~cm}^{2} \mathrm{~s}$ [15], and $\alpha_{e}<\alpha_{a}$ ), we can assume $a_{e} \frac{\partial^{2} T_{e}}{\partial z^{2}}, a_{a} \frac{\partial^{2} T_{e}}{\partial z^{2}}=0$, and the function $\frac{a_{e} h v}{c_{e}} \cdot J(t, z)$ can be replaced with the function $\Gamma \delta(z-v t)$, describing the heat source caused by the fast absorption of the radiation in the medium. The solution can be derived in the form:

$$
\begin{aligned}
& T_{e}(t)-T_{e}(0)=\int_{0}^{t}\left(A+(1-A) \exp \left(-\lambda\left(t-t^{\prime}\right)\right)\right) \\
& \Gamma \delta\left(z-v t^{\prime}\right) d t^{\prime} \\
& T_{a}(t)-T_{a}(0)=\frac{1}{\tau_{e p} \cdot c_{e}} \int_{0}^{t}\left(1-\exp \left(-\lambda\left(t-t^{\prime}\right)\right)\right) \\
& \Gamma \delta\left(z-v t^{\prime}\right) d t^{\prime}
\end{aligned}
$$

where $A=\frac{1}{C_{e}^{2}}+\frac{1}{C_{e}+C_{a}}, \lambda=\frac{1}{\tau_{e p}}\left(\frac{1}{c_{e}}+\frac{1}{c_{a}}\right)$.

\subsection{Results}

By means of Monte Carlo numerical simulation of propagation of radiation through the skin tissues, we obtained the intensity distributions over the tissue depth (Fig. 2). The calculation approximated the solution of the radiation transfer equation by modelling all possible photon trajectories passing through the skin tissue model. The steady-state form of the radiation transfer equation in a homogeneous medium can be written as the integral equation $[16,17]$ :

$$
\begin{gathered}
L(r, s)= \\
\int_{R-0}^{\infty} \exp \left(-\mu_{t r} R\right) \mu_{s} \int_{4 \pi} p\left(s, s^{\prime}\right) L\left(r-R s, s^{\prime}\right) d \Omega^{\prime} \mathrm{dR}+ \\
+\int_{R-0}^{\infty} \exp \left(-\mu_{t r} R\right) Q(r-R s, s) d R
\end{gathered}
$$

where $\mathrm{L}$ is the luminance at the point $\mathrm{r}$ in the direction $\mathrm{s}$, considered for both the light source and for the radiation scattered towards the direction s; R is the path length, Q is the light emitted in the direction $s$ by the source at the point $\mathrm{r}-\mathrm{Rs}$. The parameter $\exp \left(\mu_{t r} R\right)$ determines the radiation transfer from the point $\mathrm{r}-$ Rs to the point $\mathrm{r}$. Thus, the first term in the equation characterises the light scattered at the point $\mathrm{r}-\mathrm{Rs}$, from any direction s' to the direction s, reaching the point $r$, and the second term describes the light emitted from the point $r-$ Rs in the direction $\mathrm{s}$.

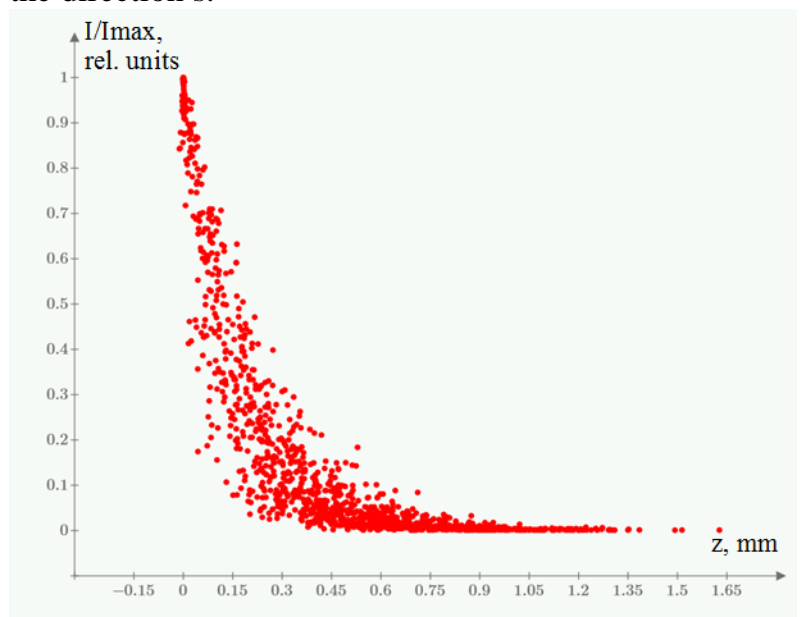

Fig. 2 Dependence of the radiation intensity upon the penetration depth (for $\lambda=800 \mathrm{~nm}$ ).

From Fig. 2 one can see that at the depth of $0.3 \mathrm{~mm}$ the radiation intensity is less than $30 \%$ of the initial level. It is known that the probability of multiphoton processes is directly proportional the second, the third and higher powers of the laser intensity. Thus, the main nonlinear processes will take place in the upper skin layer, the epidermis.

The dynamics of the electron system can be described by the energy distribution. Figure 3 presents the energy distribution of electrons depending on the time passed after the pulse impact. The state of the 
electron subsystem is shown immediately after the impact (a), after 1 ps, when a part of electrons has transferred their energy to the atom subsystem as a result of relaxation (b), and after more than $3 \mathrm{ps,} \mathrm{when}$ the most part of the electrons has transferred their energy to the atom subsystem, and the entire system tends to thermal equilibrium (c).

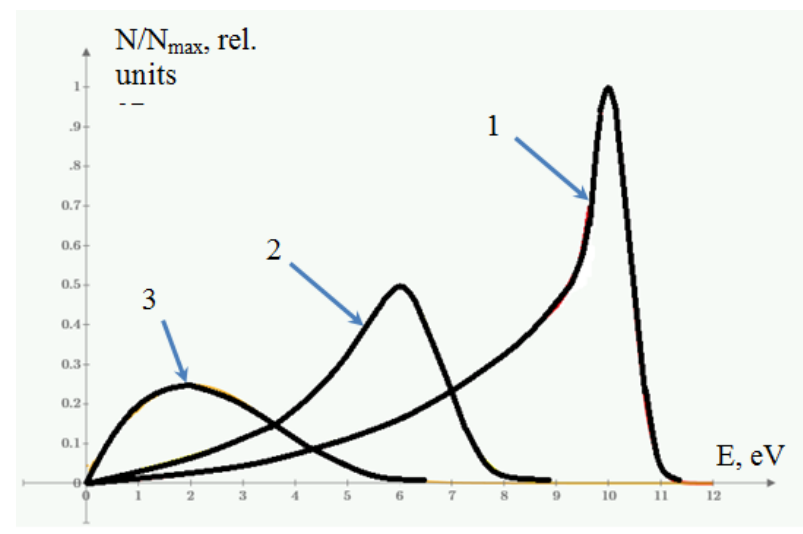

Fig. 3 Distribution of electrons over the energy levels depending upon time: 1 - the energy transfer to the electron subsystem, 2 and 3 - partial relaxation of electrons and transfer of the energy to the atomic subsystem.

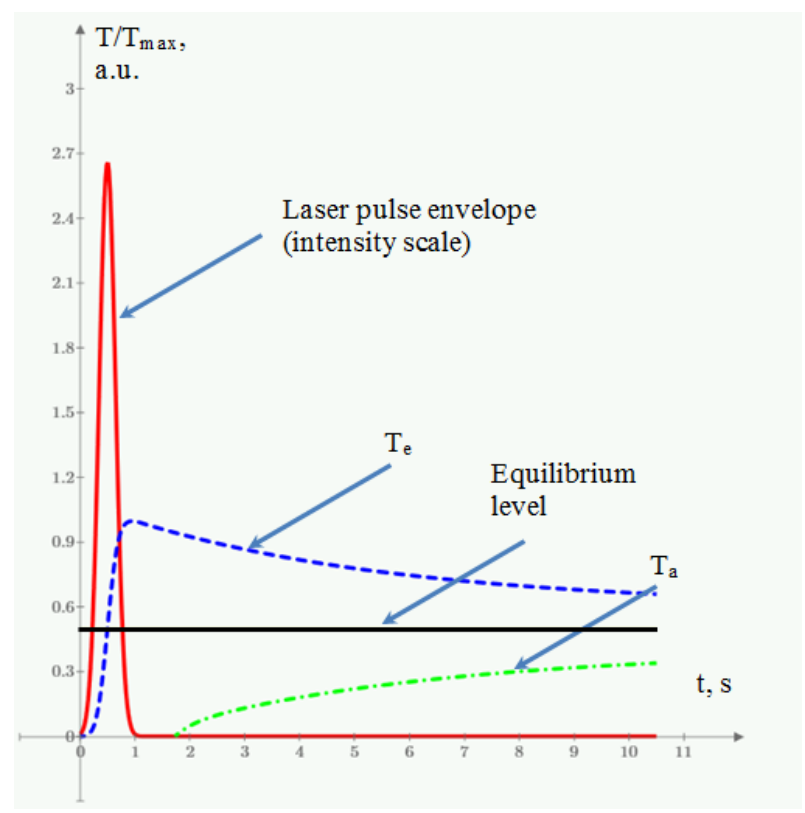

Fig. 4 Thermodynamics of the system. The laser pulse, dynamics of the electron gas temperature $T_{e}$ and the crystal lattice temperature $T_{a}$.
The absorption of light quanta by nonequilibrium electrons of the dielectric increases their kinetic energy. This leads to the growth of electron temperature during the light pulse, whereas the lattice remains practically cold. Therefore, one can divide the process of the action of the femtosecond laser radiation on the skin into three stages. At the first stage during the femtosecond pulse the multiphoton excitation of water molecules occurs. The energy of ionisation in this process amounts to 6.5 $\mathrm{eV}$, so that to ionise one water molecule nearly 5 photons $(800 \mathrm{~nm})$ are necessary, which is a cause for the reduction of the quantum efficiency $(\eta \leq 20 \%)$. Simultaneously the process of impact ionisation takes place. As a result, before the end of the laser pulse action $\mathrm{N}_{\max } \approx \eta \cdot \mathrm{E} / \mathrm{hw}$ ( $\mathrm{E}$ being the pulse energy) electrons will be excited to the upper ionised states of the water molecules with the energy above $U_{i}=6.5 \mathrm{eV}$. The second stage occurs after the action of the femtosecond pulse and lasts until the complete transfer of the energy from the electrons to the phonon subsystem. The electron gas is cooled and the collagen molecules are heated (tens of picoseconds). The third stage is the distribution of heat over the bulk sample (a few microseconds).

\section{Conclusion}

We presented a mathematical model of the propagation of femtosecond radiation through the cutaneous covering. Using the Mote Carlo numerical simulation, we determined the dependence of the radiation intensity upon the penetration depth. By the aid of the analytical solution of the system of equations describing the energy balance between the electron subsystem and the atomic one, we considered the mechanism of the effect of femtosecond radiation on the skin and estimated the temporal processes that occur in the course of the femtosecond radiation acting on the skin.

\section{Acknowledgments}

The results of this work were obtained within the framework of the State order №3.1675.2014/K from the Ministry of Education and Science of Russian Federation.

We also express our gratitude to Dr. Sergey A. Chivilikhin, Cand. Sci, staff member of the Photonics and Optoinformatics Chair of the ITMO University (Saint-Petersburg), for consultations and essential contribution to this work. 